
Sinter bem Sađregijter befinbet fiđ ein augfübrlides Derzei(hnis ber

\section{Guttentagfden $\Xi_{\mathfrak{a}} \mathfrak{m} \mathfrak{m} \mathfrak{n} \mathfrak{g}$ Deutider Reich : und Preüijher Bejeze}

\section{- Zertaugagaben mit Mnmerfungen; Zajめenformat -,}

Die alle widtigeren (Befege in unbebingt au. verläffigem aborud und mit muftergultiger Erläuterung wiebergibt. 


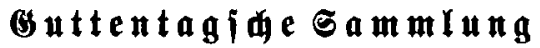

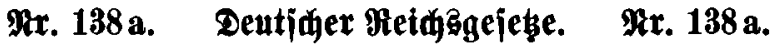

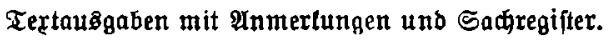

\title{
Arbeiterjobus und arbeitorecht
}

1. $\mathfrak{A} \mathfrak{a} f \mathfrak{a} \mathfrak{g} e$ bea $\operatorname{bet} \in \tan$

Dr. 2loolf Günţer.

\author{
2. 2luflage \\ 1. Teil: \\ 2lrbeitszeitichuł
}

Das Arbeitazeitnotgejes unb bie librige arbeitbzeitred.

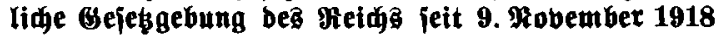

$$
\text { er } \mathfrak{l} a ̈ \mathfrak{u} \text { e } \mathfrak{x} \mathfrak{t} \mathfrak{v} \circ \mathfrak{n}
$$

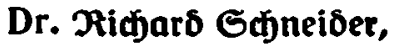

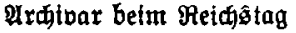

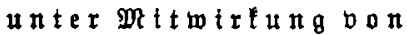

Dr. 2rbolf Günther,

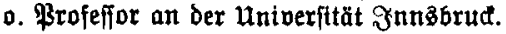

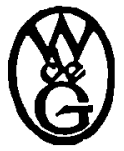

Berlit und letpzig 1927.

\section{Wolter de Gruyter \& $\mathcal{C o}$.}

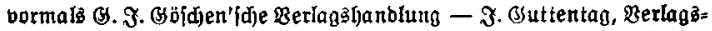
bufhandung - Beorg Betmer - \$arl 3. Trübner - Bett \& Comp. 
